\title{
Image Enhancement by High-Order Gaussian Derivative Filters Simulating Non-classical Receptive Fields in the Human Visual System
}

\author{
Kuntal Ghosh, Sandip Sarkar, and Kamales Bhaumik \\ Microelectronics Division, Saha Institute of Nuclear Physics, \\ 1/AF Bidhannagar, Kolkata-64, India \\ kuntal.ghosh@saha.ac.in
}

\begin{abstract}
The non-linearity exhibited by the non-classical receptive field in human visual system has been combined with the linear classical receptive field model. This enables us to construct higher order Gaussian Derivatives as a linear combination of lower order derivatives at different scales. Based on this, a new kernel which simulates nonclassical receptive fields with extended disinhibitory surrounds, has been proposed. It is easy to implement and finds justification from an old psychophysical angle too. The proposed kernel has been shown to perform better than the well-known Laplacian kernel, which models the classical excitatory-inhibitory receptive fields.
\end{abstract}

\section{Introduction}

David Marr [1] introduced the Laplacian of Gaussian (LOG) kernel for the purpose of edge detection and demonstrated the equivalence of this operator to the classical centre-surround Difference of Gaussian (DOG) [2 3] based receptive field model in Human Visual System (HVS). The reason behind Gaussian smoothing prior to differentiation (Laplacian in this case), as is well known in Computer Vision, is to well-pose an ill-posed problem [45. But even a simple Laplacian kernel, used for the purpose of image enhancement [6], may also be looked upon as a mimetic representation of the excitatory-inhibitory mechanism of visual receptive field. In this paper we are going to incorporate a physiologically reported disinhibitory mechanism 7$] 8$ into the classical excitatory-inhibitory DOG model of visual receptive field, by including two additional Gaussians with larger variances representing an extended surround to the classical receptive field. It has been shown [9] with the help of a theorem by Ma and Li [10] that the DOGLOG equivalence can be proved through a more rigorous approach as compared to that of Marr [1] and can also be generalized for any number of multi-scale Gaussians. As a continuation of this approach we have, in this paper, been able to express a sixth order derivative of Gaussian as a sum of second and a fourth order derivative in any scale ratio. This has enabled us to construct a generalization of the traditional Laplacian kernel that has been found to exhibit better performance for image enhancement. 


\section{The Proposed Model}

It is well-known that DOG model of receptive field is the classical model for the centre-surround antagonistic effects observed in HVS. However physiological experiments 7] also lend support to the existence of an extended disinhibitory surround that is also capable of modulating the behaviour of the cell in question in a non-linear fashion. Though there is confusion regarding the polarity of this extended surround, recent experiments 8] suggest the existence of both positive as well as negative sub-units in this region. We are now going to show that such a scheme fits quite rigorously into Ma-Li's theorem [10] of construction of higher order Gaussian Derivatives which are a likely possibility in HVS according to the works of Young [1] and Koenderink [12.

Ma-Li's theorem states that any $2 k$ th order derivative filter which is generally a $2 k$ th order derivative of an analytical smooth function derivable up to $2 k$ th order, can be expressed as a weighted sum of $k+1$ components equal to an even function at different scales, the weights depending upon the corresponding scale ratios. In other words if $g$ is the primitive Gaussian filter, then any $2 k$ th order derivative filter $h_{2 k}(x)$ may be expressed as:

$$
\begin{aligned}
h_{2 k}(x) & =\sum_{j=0}^{k} \frac{\alpha_{j}}{\sigma_{j}} g\left(\frac{x}{\sigma_{j}}\right), \\
\text { where, } g\left(\frac{x}{\sigma}\right) & =\frac{1}{\sqrt{2 \pi}} e^{-\frac{x^{2}}{2 \sigma^{2}}}
\end{aligned}
$$

In order that $h_{2 k}(x)$ may represent a derivative filter, the following conditions need to be satisfied:

$$
\begin{aligned}
\alpha_{0}+\alpha_{1}+\alpha_{2}+\cdots+\alpha_{k} & =0 \\
\alpha_{0} \sigma_{0}^{2}+\alpha_{1} \sigma_{1}^{2}+\alpha_{2} \sigma_{2}^{2}+\cdots+\alpha_{k} \sigma_{k}^{2} & =0 \\
\vdots & \\
\alpha_{0} \sigma_{0}^{2 k}+\alpha_{1} \sigma_{1}^{2 k}+\alpha_{2} \sigma_{2}^{2 k}+\cdots+\alpha_{k} \sigma_{k}^{2 k} & =\frac{(2 k) !}{m_{g, 2 k}}
\end{aligned}
$$

and if the matrix $M_{\sigma}$ where

$$
M_{\sigma}=\left[\begin{array}{cccc}
1 & 1 & \cdots & 1 \\
\sigma_{0}^{2} & \sigma_{1}^{2} & \cdots & \sigma_{k}^{2} \\
\vdots & & & \\
\sigma_{0}^{2 k} & \sigma_{1}^{2 k} & \cdots & \sigma_{k}^{2 k}
\end{array}\right]
$$

is non-singular, and $m_{g, 2 k}$ represents the $2 k$ th order moment of the function $g(x)$.

Using the above conditions it can be shown [9] that for a fourth order derivative filter we have:

$$
\begin{aligned}
\alpha_{0} & =c\left(\sigma_{2}^{2}-\sigma_{1}^{2}\right) \\
\alpha_{1} & =-c\left(\sigma_{2}^{2}-\sigma_{0}^{2}\right) \\
\alpha_{2} & =c\left(\sigma_{1}^{2}-\sigma_{0}^{2}\right)
\end{aligned}
$$

where $c$ is a constant. 
The above equations clearly show that a fourth order derivative filter, thus designed out of three Gaussian filters at different scales, is inherently non-linear. This is because the co-efficients of each of these Gaussian filters is a function of the scales of the other two, which is not the case in case of the DOG equivalence to LOG, where the two coefficients are simply equal to one another 10. It is however difficult to ascertain whether this non-linearity is exactly akin to the non-linearities observed in the non-classical receptive field, since the nature of the non-linearity in the physiological system is yet to be properly identified. But there are some close similarities of the above model with the physiological system. Polarities of the three Gaussians, defined through equations (3) to (5) indicate the possibility of considering the three Gaussians as representatives of the centre, surround and disinhibitory extended surround portions of the non-classical receptive field respectively. Now in the limit of a large extended disinhibitory surround, evidences of which exist in physiology [8], it is possible to apply a limiting condition to the smallest of the three variances, which represents the excitatory centre, so that $\sigma_{0} \rightarrow 0$, whereby :

$$
h_{4}(x, \sigma)=m h_{2}\left(x, \sigma^{\prime}\right)+h_{2}\left(x, \sigma^{\prime \prime}\right)
$$

where $\sigma^{\prime}$ and $\sigma^{\prime \prime}$ are two arbitrary scales and $m$ is an amplitude scale factor.

So in two dimensions:

$$
\nabla^{4} G(r, \sigma)=m \nabla^{2} G\left(r, \sigma^{\prime}\right)+\nabla^{2} G\left(r, \sigma^{\prime \prime}\right)
$$

Following the same procedure for a sixth order derivative, we find that two additional Gaussians represent contributions from the positive and negative subunits in the extended surround. It has also been shown 9] that the two new Gausssians in order to satisfy the derivative condition do indeed come in opposite polarity. Thus in two dimensions we arrive at:

$$
\nabla^{6} G(r, \sigma)=m \nabla^{2} G\left(r, \sigma^{\prime}\right)+n \nabla^{2} G\left(r, \sigma^{\prime \prime}\right)+\nabla^{2} G\left(r, \sigma^{\prime \prime \prime}\right)
$$

for two amplitude scale factors $m$ and $n$, and another arbitrary scale $\sigma^{\prime \prime \prime}$ so that with the help of equation (7) we finally arrive at:

$$
\nabla^{6} G(r, \sigma)=m \nabla^{2} G\left(r, \sigma^{\prime}\right)+\nabla^{4} G\left(r, \sigma^{\prime \prime \prime \prime}\right)
$$

where the final two scales are designated as $\sigma^{\prime}$ and $\sigma^{\prime \prime \prime \prime}$.

We thus arrive at a new, more general model for the Human Visual System that accounts for both linear, classical as well as non-linear, non-classical receptive fields in terms of even-order derivatives of Gaussian, up to the sixth order. Following the methodology described above, the eighth and the tenth order Gaussian Derivatives can also be similarly designed.Thus, through a multi-scale combination of various smoothing layers of neurons in retina and LGN, even order Gaussian Derivatives starting from the second order up to at least the tenth order can definitely be computed in visual cortex. The present model therefore justifies the experimental and theoretical findings of Young [1] and Koenderink [12] who also suggest the existence of Gaussian Derivatives up to the order ten in HVS. 


\section{Results and Discussion}

The advantage of expressing a sixth order Gaussian derivative as a linear combination of a second order and a fourth order one at two different possible scales may be easily understood. Visual processing operates through a very flexible methodology that always changes according to the situation. By changing the value of $m$ and operating at various combinations of scales, that may be dealt with a top-down approach, the visual system is capable of functioning bottom-up by taking advantage of both the classical as well as the non-classical receptive field. It has already been shown [13] that such a tuning of scale is capable of explaining many low-level brightness-contrast illusions as perceived by HVS. In figure 1, we are only giving two examples where such a combination clearly surpasses the standard LOG filter in terms of both enhancement and localization capability. In the first case the value of $m$ is 0.5 , which implies that the fourth order derivative is more dominant as compared to the second order one. In the second case, the value of $m$ is 9 , which implies that the fourth order derivative is less dominant as compared to the second order one.

As early as in 1868, the great physicist E. Mach provided a psychophysical model of visual computation [14] where he claimed that visual response is determined through a combination of absolute light intensity and its Laplacian. The present model for any order of derivative reduces to Mach's model if the scale of the higher derivative goes to zero in the limit i.e. in the present case if $\sigma^{\prime \prime \prime \prime} \rightarrow 0$. Using finite difference approximation theorem, it has been shown [15] that for $m=9$, it is possible to construct a new kernel for the purpose of image enhancement that far exceeds the traditional Laplacian kernel in performance. If we take a look at the two kernels given below,
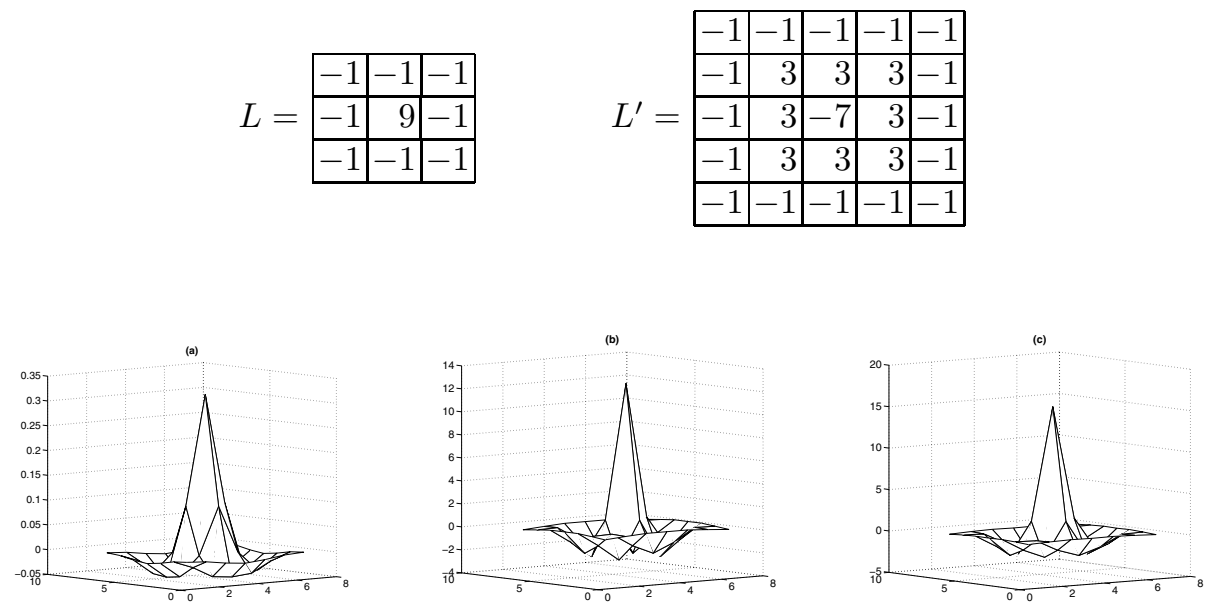

Fig. 1. (a) A Laplacian of Gaussian Mask (b) A combination of Laplacian and Bilaplacian at $m=0.5$ (c)A combination of Laplacian and Bi-laplacian at $m=9$. Variances have been fixed at unity in all the cases for the sake of comparison. 
(a)

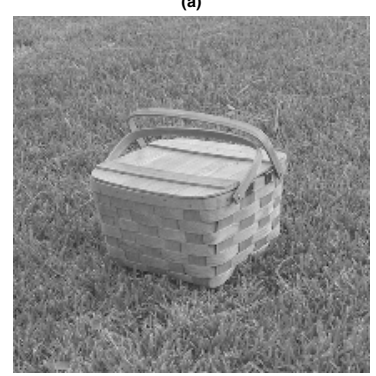

(a)

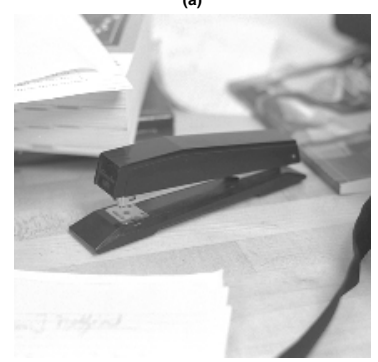

(b)

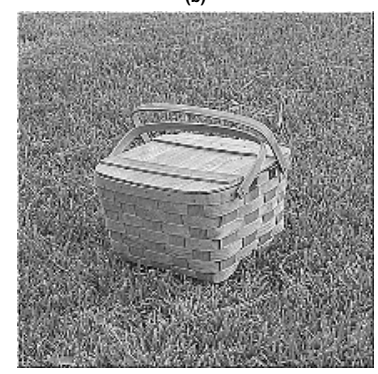

(b)

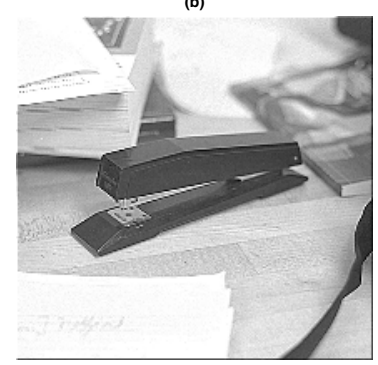

(c)

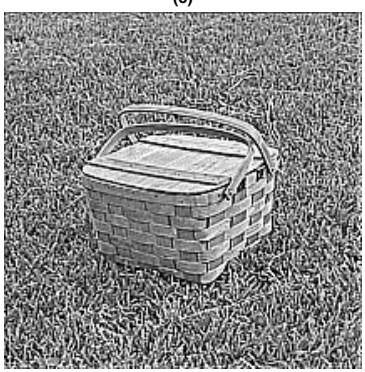

(c)

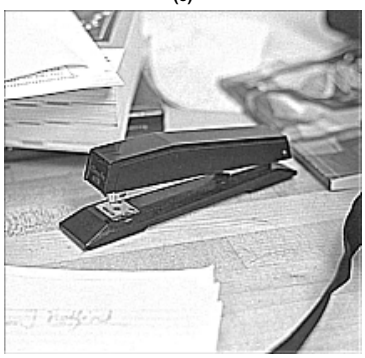

Fig. 2. (a) Two images of basket and stapler (b) the images enhanced with the Laplacian-based kernel (c) the images enhanced with the kernel derived from the proposed methodology

it is easy to see from the polarities of the weight factors that while the one constructed out of Laplacian $(L)$ may clearly represent the classical excitatoryinhibitory model, the other one $\left(L^{\prime}\right)$ represents the non-classical excitatoryinhibitory-disinhibitory model of receptive field. In figure 2, we show the results of convolving two images with $L$ and $L^{\prime}$ kernels. The results clearly show that the new kernel $L^{\prime}$ outperforms the Laplacian based kernel $L$ in terms of image enhancement. The parameters of $m$ that have been chosen here are certainly not unique. It can be easily verified that one shall get similar results for other values as well.

\section{Acknowledgement}

We thank Mr. Ratan kumar Saha, Mr. Sankhasubhra Nag, Mr. Arunabha Adhikary and all members of Microelectronics Division, SINP for their help and cooperation.

\section{References}

1. Marr, D., Hildreth, E.: Theory of edge detection. Proceedings of Royal Society of London B 207 (1980) 187-217

2. Rodieck, R. W.: Quantitative analysis of cat retinal ganglion cell response to visual stimuli. Vision Research 5 (1965) 583-601 
3. Enroth-Cugell, C., Robson, J. G.: The contrast sensitivity of the retinal ganglion cells of the cat. Journal of Physiology (London) 187 (1966) 517-552

4. Poggio, T., Torre, V.: Ill-posed problems and regularization analysis in early vision. MIT AI Memo 773 (1984)

5. Poggio, T. Voorhees, H., Yuille, A.: A Regularized solution to edge detection. MIT AI Memo 833 (1985)

6. Gonzalez, R. C., Woods, R. E.: Digital image processing. Second Ed. Third Indian Reprint, Pearson-Education (2003) 125-131

7. Ikeda, H., Wright, J.: Functional oganization of the periphery effect in retinal ganglion cells. Vision Research 12 (1972) 1857-1879

8. Passaglia, C. L., Enroth-Cugell C., Troy J. B.: Effects of remote stimulation on the mean firing rate of cat retinal ganglion cells. Journal of Neuroscience 21 (2001) $5794-5803$

9. Ghosh, K., Sarkar, S., Bhaumik K.: A bio-inspired model for multi-scale representation of even order gaussian derivatives. Proceedings of International Conference on Intelligent Sensors, Sensor Networks and Information Processings (ISSNIP), IEEE EX 994 ISBN: 0-7803-8893-3 (2004) 497-502

10. Ma, S. D., Li, B.: Derivative computation by multiscale filters. Image and Vision Computing. 16 (1998) 43-53

11. Young, R. A.: The gaussian derivative theory of spatial vision: analysis of cortical cell receptive field line weighing profiles. GMR-4920 (1985)

12. Koenderink, J. J., van Doorn, A. J.: Receptive field families. Biological Cybernetics 63 (1990) 291-297

13. Ghosh, K., Sarkar, S., Bhaumik, K.: Low-level brightness contrast illusions and nonclassical receptive field of mammalian retina. Proceedings of Second International Conference on Intelligent Sensing and Information Processing (ICISIP), IEEE EX 979 ISBN: 0-7803-8840-2 (2005) 529-534

14. Mach, E.: On the physiological effect of spatially distributed light stimuli (1868). Mach Bands: Quantitative Studies On Neural Network In The Retina, F. Ratliff (Ed), Holden-Day, San Francisco (1965) 299-306

15. Ghosh, K., Sarkar S., Bhaumik, K.: A new mask for unsharp masking based on human visual system. Proceedings of National Conference on Image Processing (NCIP), IEEE Conf. Rec. 10435 (2005) 113-116 